\title{
Experimental set-up and efficiency evaluation of zero-field-cooled (ZFC) YBCO magnetic bearings
}

\author{
A.J. Arsénio, M.V. Carvalho, C. Cardeira, Member, IEEE, R. Melício, Senior Member, IEEE, \\ P.J. Costa Branco, Senior Member, IEEE
}

\begin{abstract}
This paper presents the conception, simulation and experimental evaluation of magnetic bearings based on $\mathrm{NdFeB}$ magnets and zero-field-cooled (ZFC) YBCO bulk superconductors to replace the bearings of a standard electric machine. Advanced research on HTS magnetic levitation has been carried out for the construction of large scale flywheels or for industry applications. However, those approaches used fieldcooled (FC) superconductors, whereas the approach shown in this paper uses ZFC superconductors. Actually, as previously shown by the authors, ZFC implementation presents much less AC losses. The approach in our horizontal flywheel research follows the ZFC-maglev structure, where the linear track was closed into a circular one with the same diameter of the superconductor magnetic bearing. The conception and geometric placement of magnets and superconductors was designed to keep symmetry along the rotating axis and minimizing air gaps. A 3D finite element model was built for simulation and validation of the effectiveness of the solution. Experimental validation was then achieved measuring the balanced and unbalanced rotor lifting and guiding forces.
\end{abstract}

Index Terms - High-temperature superconductors; magnetic bearings; magnetic levitation; Superconducting machines, rotors;

\section{INTRODUCTION}

When type-II superconductor bulks are cooled in presence of a magnetic field, known as the field cooling technique, they trap and memorize that magnetic field. After FC they perform as permanent magnets (PMs), generating a magnetic field equal to the trapped one. When they are located in a magnetic field different to the trapped field, they get attracted to a position where the existing field almost equals the trapped field [1,2]. Contrary, the zero-field-cooling technique consists in cooling a superconductor in the absence of magnetic fields. When a ZFC superconductor bulk is positioned in the middle of an existing magnetic field, it is repealed to a position where the magnetic flux is nearly zero[3,4]. Majority of existing levitation systems use PMs on one part and FC superconductor bulks on the other part, being used as frictionless rotating bearings that can be horizontal or vertical axis rotating [5-8].

This work was supported by FCT, through IDMEC, under LAETA, project UID/EMS/50022/2013 and under project PTDC/EEEI-EEL/4693/2014 HTSISTELEC

A.J. Arsénio, C. Cardeira (Corresponding author) and P. Branco are with IDMEC, Instituto Superior Técnico, Universidade de Lisboa, Lisboa, Portugal (e-mails: \{antoniojcosta, carlos.cardeira, pbranco\}@tecnico.ulisboa.pt).

M. Carvalho is with Instituto Superior Técnico, Universidade de Lisboa, Lisboa, Portugal (e-mail: martimvazdecarvalho@gmail.com).

R. Melício is with Departamento de Física, Escola de Ciências e Tecnologia, Universidade de Évora and IDMEC, Instituto Superior Técnico, Universidade de Lisboa, Portugal (e-mail: ruimelicio@gmail.com).
In $[9,10]$ frictionless superconductor magnetic bearing (SMB) systems studied were designed to levitate based on the FC technique. This implies significant hysteresis losses due to the magnetic flux trapping. In [11], to drop them, it was proposed a linear electromagnetic launcher with propulsion forces using Meissner effect based on ZFC technique. In $[12,13]$, it was proposed a linear HTS magnetic levitation system based on the ZFC technique with guidance. The guidance was obtained by an adequate distribution of existing magnetic fields, generated by specific array configurations of multi-pole permanent magnets and its coupling with a ZFC superconducting structure.

Taking into account that context, the main contribution of this paper is the viability analysis of a new ZFC-based and horizontal SMB made of HTS bulks and PMs. Specific magnetic circuit geometry is proposed and simulated. At last, a prototype of the ZFC SMB was constructed to evaluate the methodology.

\section{The ZFC MAgnetic BeAring Design}

The ZFC-SMB is composed by a fixed part (stator) and a rotating but levitating part (rotor), as shown in Fig. 1. The stator contains 2 discontinuous rings of 16 equally spaced bulk YBCO superconductors. The inner rotor part contains 3 discontinuous rings with 5 equally spaced $\mathrm{NdFeB}$ permanent magnets each one.

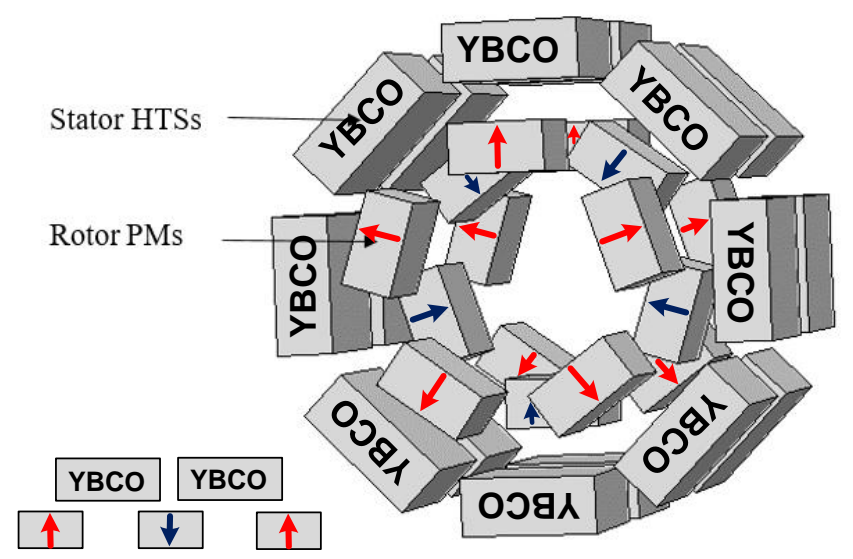

Figure 1. ZFC-SMB: proposed magnetic circuit design.

The three inner discontinuous rings of PMs in Fig. 1 are magnetized in alternate such as the two border PMs rings have concordant polarizations and the middle ring of magnets opposite polarization. The discontinuous rings of PMs and HTS bulks are interposed in such a way to provide guidance forces [1]. Simulation results in Fig. 2 were achieved using a 
finite element modeling (FEM) approach. Each PM $\left(B_{r}=1.2 \mathrm{~T}\right)$ has rectangular form with $25 \times 25 \times 12 \mathrm{~mm}$ and $0.06 \mathrm{~kg}$. Each YBCO has also a rectangular form with $32 \times 32 \times 14 \mathrm{~mm}$ and $0.09 \mathrm{~kg}$.

Concerning the YBCOs model, in this design evaluation one only has used a relative magnetic permeability of $\mu_{r}=0.2$. Why this value and why it allowed us to achieve enough precise conclusions? As previously verified by us in [11], to the point of view of a magnetic circuit, the YBCO from "CAN Superconductors" gave force values with good precision relative to the measured ones when using $\mu_{r}=0.2$. Notice however that with this value, some degree of flux pinning exists. Also, this value also has been confirmed in other experiments in our laboratory when using the YBCOs as magnetic screens. For computation of joule losses and associated temperature increase in the superconductors, this approach is far from be used. That has been recently solved in our group in [2] using an electro-thermal YBCO model developed in Comsol Multiphysics framework by using its differential partial equations' input and associated solver. The $\mathrm{H}$-formulation was used by us as was in [9] but now coupled with the thermal physics of the superconductor. Next step will employ that electro-thermal model in the 3D model of the bearing. Therefore, using such simple model allowed us, at this level, to test the proposed SMB design not only without excessive computational effort due its 3D representation, but also with enough reliable quantitative results as is shown follow.

Fig. 2-left shows the side view of the 3D FEM model implemented. It shows the PMs polarization and a yellow cross line 'A-A' used to visualize the flux lines in its axis plane, as Fig. 2-right shows.

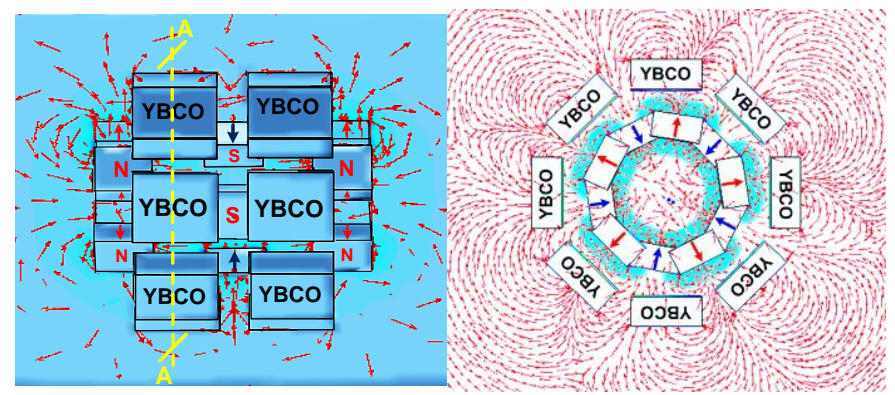

Figure 2. ZFC-SMB: left shows side view of the 3D FEM model and the cross line 'A-A' used to visualize the flux lines in its axis plane displayed at right.

\section{A. Lifting and Guidance Rotor Forces}

Fig. 3 shows in a red dotted line the rotor lifting force vs. rotor eccentricity obtained from the 3D-FEM model and its 16 HTSs. Results were obtained only decentering the rotor between a range of $\pm 10 \mathrm{~mm}$. Guidance forces are shown in Fig. 4 only for some positive eccentricities. For the current geometric dimensions, the horizontal displacement interval between $\pm 10 \mathrm{~mm}$ is the stable one, where the rotor can return to its equilibrium position. Fig. 4 shows that $12 \mathrm{~mm}$ is the highest eccentricity allowed, since the maximum lifting and guidance forces must be higher than the rotor weight $(8.83 \mathrm{~N})$. Both results in Figs. 3 and 4 show that force values remain higher than the rotor weight, thus indicating that the ZFC-
SMB is viable and self-sustainable for both horizontal and vertical rotor eccentricity values.

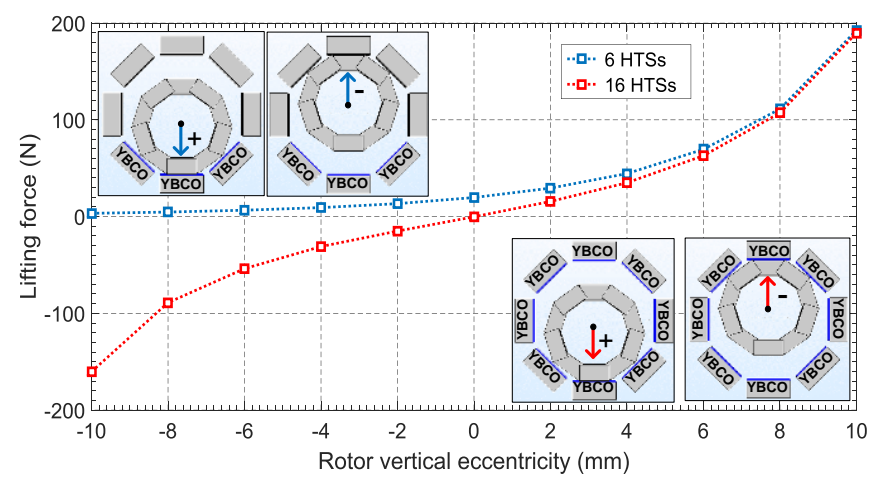

Figure 3. ZFC-SMB: red dotted line plots the lifting rotor force for different rotor eccentricity values.

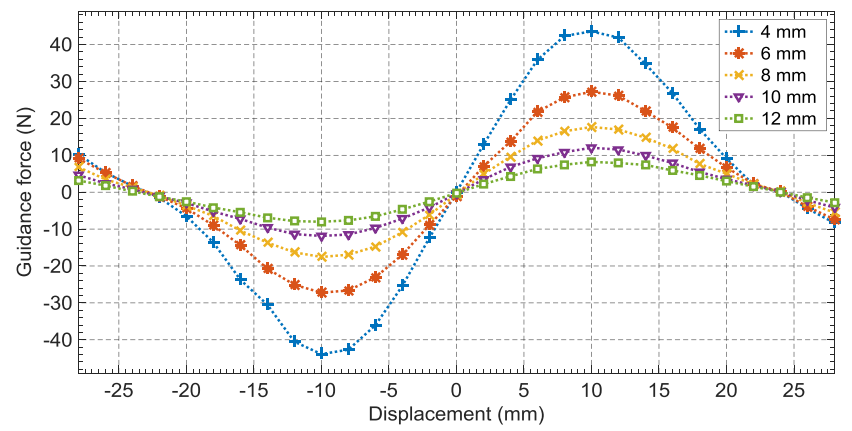

Figure 4. ZFC-SMB: balanced operation, guidance forces, different positive eccentricities.

\section{B. The ZFC-SMB Computer Aided Design Model}

The use of a 3D CAD design software for conceptual design has been widely spread. Moreover, CNC machines are nowadays able to produce real prototypes based on the $3 \mathrm{D}$ $\mathrm{CAD}$ designed models. Lately, additive manufacturing techniques like 3D printers became more and more used for rapid prototyping [15]. Hence, a SMB prototype was modelled in $3 \mathrm{D}$ to meet the following requirements: i) provide a structure that keeps the relative positions between the PMs and also between the superconductors, and also keeps uniform the stator/rotor air-gap [4]; ii) provide a sealed body to cool and maintain the entire stator and its superconductors immersed in liquid nitrogen; iii) a modular prototype development, and iv) design a prototype having an easy assembly /disassembly features for practical magnets and superconductors accessibility, maintenance and/or replacement.

\section{1) Stator block}

Fig. 5 shows the stator block in an exploded view. It supports 16 HTSs distributed in two rings made by four equal slices (this type of slice construction was used to make possible a manufacturing in a CNC milling machine). Slices are thus assembled two by two to produce the whole stator, enclosing each one a set of 8 cavities, where superconductor bulks are to be positioned. All these cavities are connected through an open channel (shown in Fig. 6), so that liquid nitrogen can flow and cool the superconductors uniformly. To combine all slices into one block, 16 holes of $6 \mathrm{~mm}$ diameter were projected, as 
indicated by the four discontinuous lines in Fig. 6. At last, liquid nitrogen must be inserted. For this, two upper holes were designed, each one for each ring. Fig. 6 also shows, in detail, one the two sets of hole' halves for the first ring.

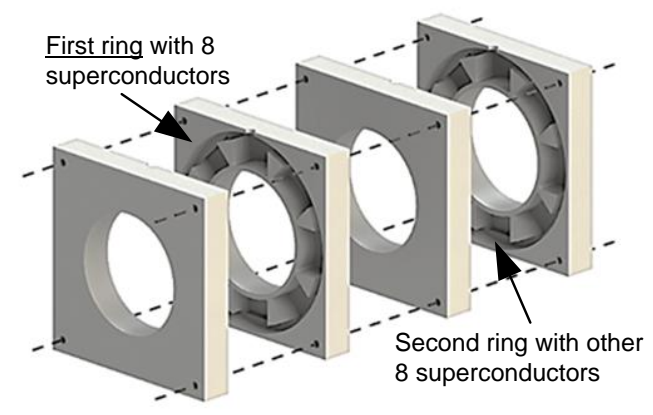

Figure 5: ZFC-SMB: stator exploded view in four slices

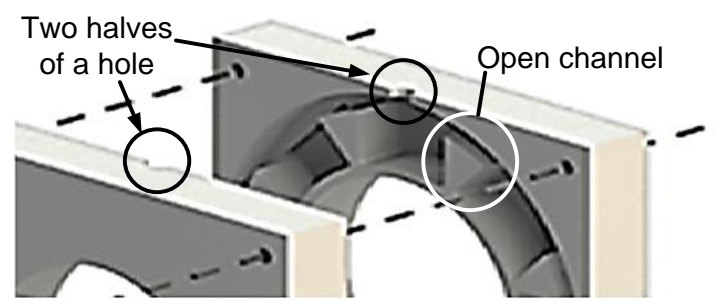

Figure 6. ZFC-SMB: detail of the open channel between the supercondutors' cavities and detail of the hole halves where the liquid nitrogen is inserted.

\section{2) Rotor block}

Fig. 7 shows an exploded figure of the rotor parts. It has two exterior rings (left and right ones), each with 5 slots for PMs with their north $(\mathrm{N})$ polarity up. The two central and complementary rotor parts in Fig. 7 will constitute the interior ring, also with 5 slots but having PMs now with south (S) polarity up. The drawing located below the rotor parts in Fig. 7 shows the relative position between the PMs that must be unaligned between them. If not, perpendicular components of the magnetic field will provoke opposite forces in the rotor as a reaction of them in the superconductors frontal surfaces.

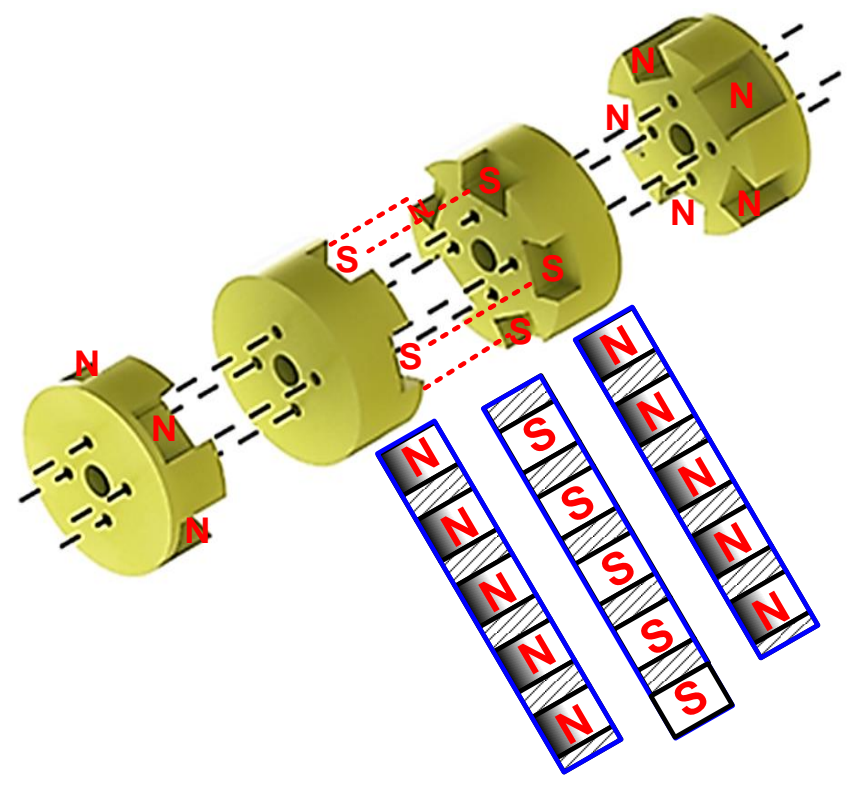

3) Virtual assembly of the prototype

Fig. 8 presents the exploded view of the complete ZFCSMB and the final assembly is shown in Fig. 9. The figure also shows that only one of the holes is used to supply the liquid nitrogen, the other one is needed to less any vapor, not allowing any pressure accumulation inside the stator block

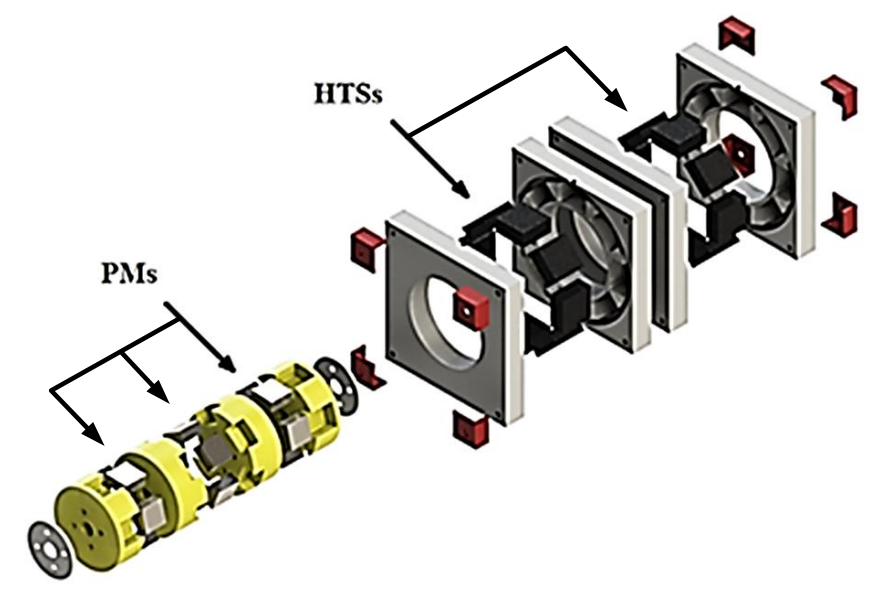

Figure 8. ZFC-SMB: exploded view of all proposed design components.

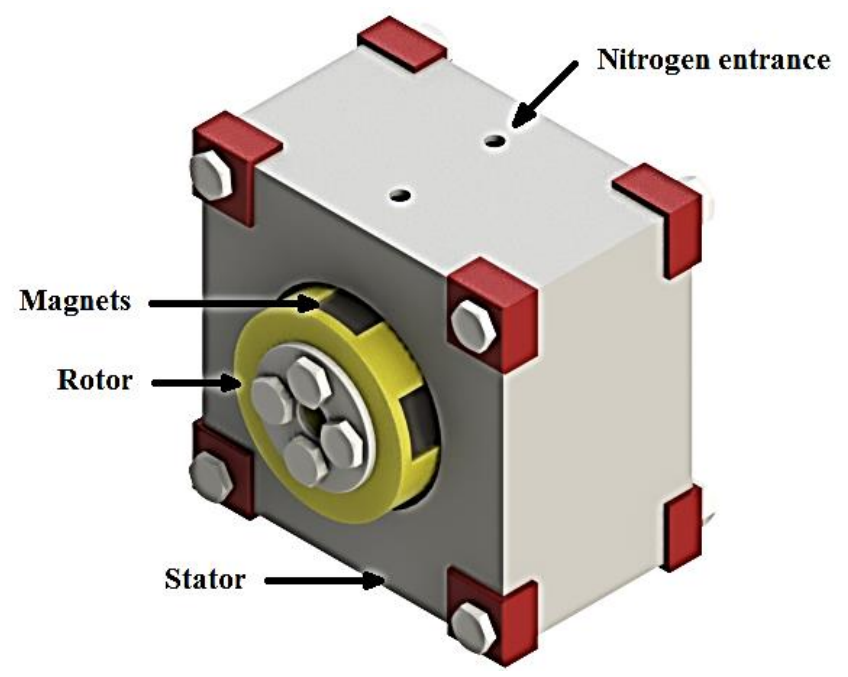

Figure 9. ZFC-SMB: final assembly view.

\section{REAL PROTOTYPE}

A real prototype of the SMB modelled using a 3D CAD Design Software was made using a CNC machine and a 3D printer. Materials were chosen to have a relative magnetic permeability of about 1 , ability to resist to temperatures in the order of $77 \mathrm{~K}$ and exhibit a low thermal conductivity coefficient. High density polyurethane was the chosen material for the stator.

For the rotor, same material was used initially but later on, as it is not submitted to such low temperatures, the usual 3D printers PLA polymer was used. Several other types of parts namely bolts, nuts, corners and washers were also made in a $3 \mathrm{D}$ printer, using in this case the ABS (AcrylonitrileButadiene-Styrene) polymer for higher resistance and lower weight. Fig. 10 shows real prototype built. See references $[16$, 17] for more details.

Figure 7: ZFC-SMB: rotor exploded view. 

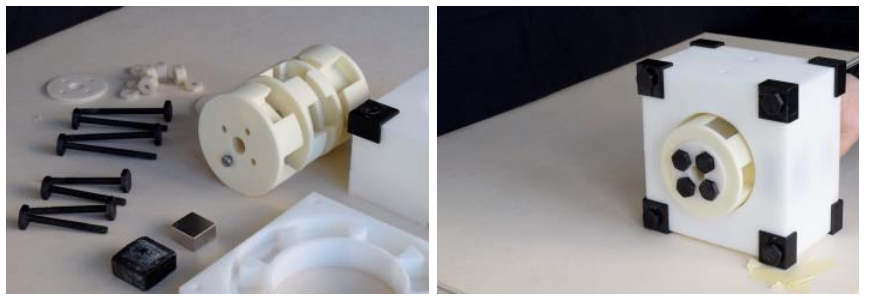

Figure 10. The ZFC-SMC prototype machined by a 3D printer.

The prototype was submitted to leak tests, which were successful after adding some silica gel between the slices and using clamps with PVC plaques to distribute the load. After that, the SMC prototype was ready for the experimental results.

\section{PROTOTYPE EXPERIMENT RESUlTS}

Due to budget limitations, namely the cost of 16 YBCO bulks, a smaller prototype version was built using only 6 YBCOs. The rotor had small changes, besides the red color (Fig.11). Each rotor slice had its thickness decreased to reduce the distance among the PM rings, increasing the lifting forces.

\section{A. Measurement of Lifting Forces}

Tests were made, all beginning with the ZFC procedure. The ZFC procedure consisted on cooling the stator without the rotor inserted. Liquid nitrogen is slowly poured to fill the stator. After a while, the whole stator is filled with liquid nitrogen, achieving the ZFC superconducting state. The rotor can be inserted in any angle. All the 15 magnets are in place so it levitates accordingly. However, due its discrete number, the equilibrium is obtained in specific angles. This fact would not happen in a continuous magnetic ring. Nevertheless, it keeps levitating when it is rotated from one angle to other angle.

Fig. 11 shows the experimental setup for measuring the lifting force. In red color, the rotor stayed lifting while the lifting force was measured by the force sensor indicated.

Before presenting the experimental results, Fig. 3 showed the simulated lifting forces for the stator using 6 HTSs. To obtain the forces, the balanced rotor and an eccentric rotor condition were also illustrated in Fig. 3. Results were displayed in the figure in a blue dotted line, revealing the high asymmetric condition between a 6 HTSs bearing and that with 16 HTSs, symmetric around its equilibrium point $(0 \mathrm{~mm})$.

Fig. 12 presents the rotor lifting forces vs rotor vertical eccentricity. Simulation is plotted using blue squares. Measured forces are plotted using red diamonds marks, together with their hysteresis effect previewed based on the results obtained by authors in [1]. The lifting forces were underestimated as shown in Fig. 12. It is clear that the physical YBCO model using $\mu_{r}=0.2$ needs to be improved, despite having the same order of magnitude and with $\mu_{r}=0.2$ some degree of flux pinning already exist.

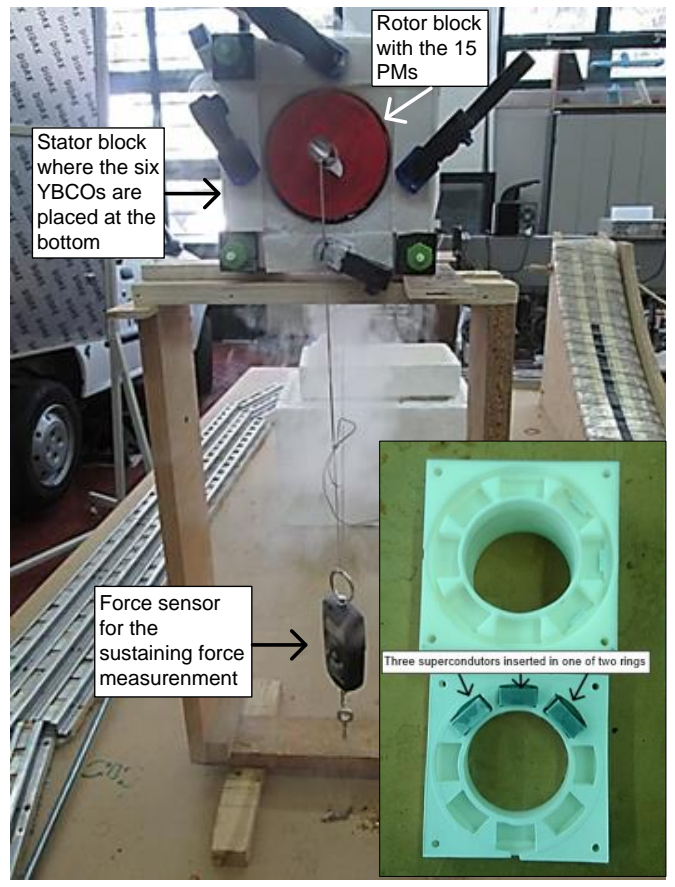

Figure 11. Experimental setup to measure the rotor lifting forces

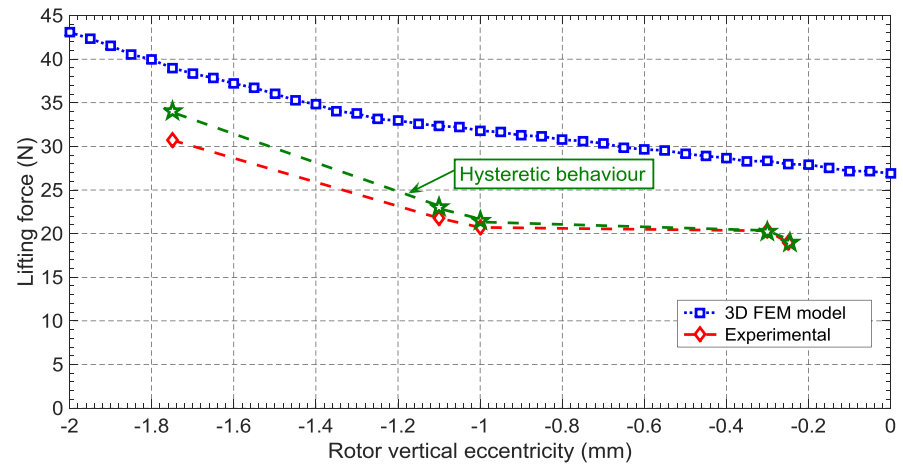

Figure 12. Rotor lifting forces vs vertical eccentricity. Blue squares: simulation. Red diamonds: measured forces. Green stars: estimated hysteresis.

\section{B. Measurement of Guidance Forces}

According to Fig 13, an axial rotor misalignment was forced to measure the guidance forces that push or pull the rotor to its axial equilibrium position. Fig. 14 shows the measured guidance forces. These were slightly higher than those projected by simulations, which can be justified again by the limited YBCO model. However, estimating the hysteresis effect on guidance forces by using similar results from [1], one verifies in Fig. 14 that forces estimated inside the stable region show very good values, near from simulated ones.

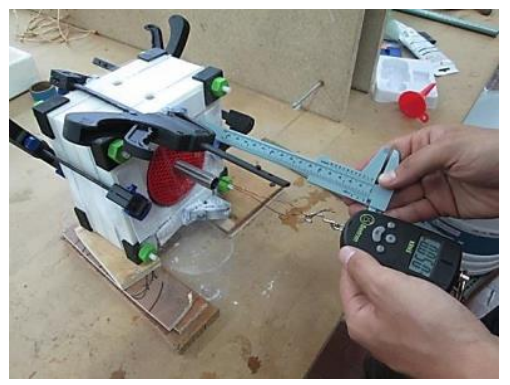

Figure 13. ZFC-SMB: guidance forces experimental setup. 


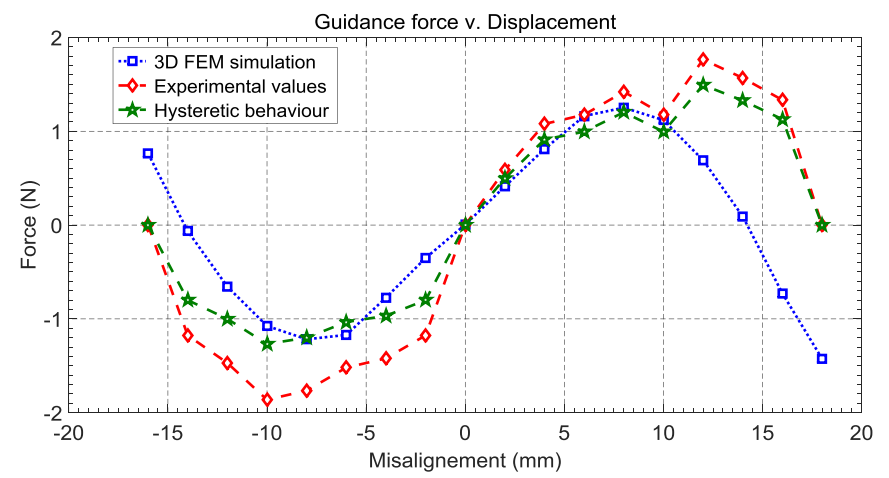

Figure 14. Rotor guidance forces vs lateral misalignment. Blue squares: simulation. Red diamonds: measured forces. Green stars: estimated hysteresis

\section{CONCLUSIONS AND FUTURE WORK}

The conception of the first ZFC-magnetic bearings for electrical machines pointed out great potentialities, even using at this point a simplified YBCO model. The lifting and guiding forces previewed by our FEM model were experimental verified with good agreement. Of course, improvements were learned during this one year research and are currently being performed by our team in the lab. Here are the most significant ones:

- a more precise YBCO modeling to not only increase the accuracy of the predicted rotor forces, but also allow us to preview Joule losses in the superconductors since they will rotate with higher speeds;

- to study the rotor's stability, a dynamic analysis of the system will be realized. Preliminary results point for a non-linear damping factor coming for energy dissipation maybe from YBCO losses (to be confirmed) and,;

- due to this, an active rotor stabilization is being implemented;

The authors like to thank the international mobility students Niels De Valck and Filip Swinnen for their useful collaboration during the initial phase of this project and Fablab EDP and Mitera for building the real prototypes.

\section{REFERENCES}

[1] B. Painho, J.A. Dente, and P.J. Costa Branco, "Superconductor losses and damping effects under zero field cooling and field cooling conditions in a HTSC-magnet levitation system," Jornal of Superconductivity and Novel Magnetism, vol. 24(1), pp. 927-937, January 2011.

[2] J. Arnaud, and P.J. Costa Branco, "Electrothermal characteristics of YBCO bulk magnets deep in $\mathrm{LN}_{2}$ : a preliminary analysis for its use as excitation system of low-speed synchronous generators," IEEE Transactions on Applied Superconductivity. vol. 26(3), April 2016.

[3] J. Zheng, Z. Deng, L. Wang, L. Liu, Y. Zhang, S. Wang, and J. Wang, "Stability of the maglev vehicle model using bulk high $T_{c}$ superconductors at low speed," IEEE Transactions on Applied Superconductivity, vol. 17(2), pp. 2103-2106, June 2007.

[4] P.J. Costa Branco, and J.A. Dente, "Design and experiment of a new maglev design using zero-field-cooled YBCO superconductors," IEEE Transactions on Industrial Electronics, vol. 59(11), pp. 4120-4127, November 2012.
[5] T.H. Sung, S.C. Han, J.S. Lee, N.H. Jeong, S.D. Hwang, and S.K. Choi, "Designs and analyses of flywheel energy storage systems using high- $\mathrm{T}_{\mathrm{c}}$ superconductor bearings," Cryogenics, vol. 42, pp. 357-362, 2002.

[6] Y.H. Han, B.J. Park, S.Y. Jung, and S.C. Han, "Study of superconductor bearings for a $35 \mathrm{kWh}$ superconductor flywheel energy storage system," Physica C: Superconductivity, vol. 483, pp. 156-161, 2012.

[7] P. Kummeth, W. Nick, and H.-W. Neumueller, "Progress in development of high capacity magnetic HTS bearings," Physica C: Superconductivity, vol. 426-431, pp. 739-745, October 2005.

[8] T. Ichihara, K. Matsunaga, M. Kita, I. Hirabayashi, M. Isono, M. Hirose, K. Yoshii, K. Kurihara, O. Saito, S. Saito, M. Murakami, H. Takabayashi, M. Natsumeda and N. Koshizuka, "Fabrication and evaluation of superconducting magnetic bearing for $10 \mathrm{~kW}$ h-class flywheel energy storage system", Physica C, vol. 426-431, pp. 752-758, 2005.

[9] F. Sass, G. G. Sotelo, R. de Andrade Junior and F. Sirois, ."Hformulation for simulating levitation forces acting on HTS bulks and stacks of $2 \mathrm{G}$ coated conductors. Superconductor Science and Technology, vol. 28, n. 12, 125012, 2015.

[10] M. Sparing, A. Berger, F. Wall, V. Lux, S. Hameister, D. Berger, M. Hossain, A. Abdkader, G. Fuchs, C. Cherif, and L. Schultz, "Dynamics of rotating superconducting magnetic bearings in ring spinning," IEEE Transactions on Applied Superconductivity, vol. 26(3), pp. 1-4, April 2016.

[11] P.J. Costa Branco, R. Almeida, and J.A. Dente, "On using Meissner effect to conceive a new linear electromagnetic launcher by zero-fieldcooling YBCO bulk superconductors," IEEE Transactions on Industrial Electronics, vol. 61(11), pp. 5894-5902, November 2014.

[12] P.J. Costa Branco, and J.A. Dente, "Design and experiment of a new maglev design using zero-field-cooled YBCO superconductors," IEEE Transactions on Industrial Electronics, vol. 59(11), pp. 4120-4127, November 2012.

[13] J. Fernandes, I. Montes, R. Sousa, C. Cardeira, and P.J. Costa Branco, "Superconductor joule losses in a zero-field-cooled (ZFC) maglev vehicle," IEEE Transactions on Applied Superconductivity, vol. PP(90), pp. 1-7, February 2016.

[14] F. Freschi and M. Repetto, "Natural Choice of Integration Surface for Maxwell Stress Tensor", IEEE Transactions on Magnetics, vol. 49, no. 5, pp.1717-1720, May 2013.

[15] K. Bassett, R. Carriveau, and D.S.-K. Ting, "3D printed wind turbines part 1: design considerations and rapid manufacture potential," Sustainable Energy Technologies and Assessments, vol. 11, pp. 186193, September 2015

[16] A.J. Arsénio, M.V. Carvalho, C. Cardeira, P.J. Costa Branco, R. Melício, "Viability of a frictionless bearing with permanent and HTS magnets", in: Proceedings of the IEEE 17th International Conference on Power Electronics and Motion Control - PEMC 2016, pp. 1231-1236, Varna, Bulgaria, 25-30 September 2016.

[17] A.J. Arsénio, M.V. Carvalho, C. Cardeira, P.J. Costa Branco, R. Melício, "Conception of a YBCO superconducting ZFC-magnetic bearing virtual prototype", in: Proceedings of the IEEE 17th International Conference on Power Electronics and Motion Control PEMC 2016, pp. 1226-1230, Varna, Bulgaria, 25-30 September 2016. 